\title{
Hidden symmetries, central charges and all that
}

\author{
B de Wit ${ }^{1}$ and $\mathrm{H}$ Nicolai ${ }^{2}$ \\ ${ }^{1}$ Institute for Theoretical Physics and Spinoza Institute, Utrecht University, Utrecht, \\ The Netherlands \\ ${ }^{2}$ Max-Planck-Institut für Gravitationsphysik, Albert-Einstein-Institut, Mühlenberg 1, D-14476 \\ Golm, Germany
}

Received 28 November 2000

Published 1 August 2001

Online at stacks.iop.org/CQG/18/3095

\begin{abstract}
In this review we discuss hidden symmetries of toroidal compactifications of 11-dimensional supergravity. We recall alternative versions of this theory which exhibit traces of the hidden symmetries while still retaining the massive KaluzaKlein states. We reconsider them in the broader perspective of M-theory which incorporates a more extended variety of BPS states. We also argue for a new geometry that may underly these theories. All our arguments point towards an extension of the number of spacetime coordinates beyond 11 .
\end{abstract}

PACS numbers: 0465, 0450, 1130P

\section{Introduction}

One of the key problems in understanding superstring theory at the non-perturbative level is the question of its fundamental underlying symmetry. Although we still do not know what this symmetry is, it has become clear in recent years that $d=11$ supergravity [1] will play a central role in this endeavour [2-4]. The hidden global symmetries arising in the dimensional reduction of this theory to lower dimensions [5] have been conjectured to also appear in Mtheory [2], albeit in a discrete version, and only for toroidal compactifications. Over the past few years it has also become clear that BPS states and supermultiplets are of essential importance in this context.

In this contribution we review the status of 'hidden symmetries' in supergravity and superstring theory, and their connection with central charges from a point of view which is somewhat different from the one usually taken. Namely, we will base our considerations on some older work $[6,7]$ where it was shown that traces of the hidden $E_{n(n)}$ symmetries of dimensionally reduced supergravity [5] remain in 11 dimensions. These considerations will lead us to conjecture the existence of effective field theories also encompassing the (nonperturbative) BPS degrees of freedom that are fully compatible with the hidden symmetries (a subset of which will be the Kaluza-Klein states). We will refer to these theories as 'BPSextended supergravities' [8], as they would be of a new type. In particular, they would live in a higher-dimensional space, such that the central charges would be associated with certain extra dimensions, in a way similar to the central charges that originate from the internal momenta 
Table 1. The hidden symmetry groups $G$ and the groups $H_{\mathrm{R}}$ for $4 \leqslant d \leqslant 9$ with the $G_{-}$ representations of the pointlike central charges. We indicate the branching into $H_{\mathrm{R}}$ representations for $d=6$ and 4 .

\begin{tabular}{llll}
\hline$d$ & $G$ & $H_{\mathrm{R}}$ & Representations \\
\hline 9 & $S L(2, \mathbb{R}) \times S O(1,1)$ & $S O(2)$ & $(\mathbf{2}, \mathbf{1}) \oplus(\mathbf{1}, \mathbf{1})$ \\
8 & $S L(3, \mathbb{R}) \times S L(2, \mathbb{R})$ & $\mathrm{U}(2)$ & $(\mathbf{3}, \mathbf{2})$ \\
7 & $E_{4(4)} \equiv S L(5, \mathbb{R})$ & $U S p(4)$ & $\mathbf{1 0}$ \\
6 & $E_{5(5)} \equiv S O(5,5)$ & $U S p(4) \times U S p(4)$ & $\mathbf{1 6} \rightarrow(\mathbf{4}, \mathbf{4})$ \\
5 & $E_{6(6)}$ & $U S p(8)$ & $\mathbf{2 7} \oplus \mathbf{1}$ \\
4 & $E_{7(7)}$ & $S U(8)$ & $\mathbf{5 6} \rightarrow \mathbf{2 8} \oplus \overline{\mathbf{2 8}}$ \\
\hline
\end{tabular}

in a Kaluza-Klein compactification. We will also argue for a 'hidden exceptional geometry' underlying $d=11$ supergravity and/or M-theory.

\section{Central charges from 11 dimensions}

In $D=11$ spacetime dimensions the anticommutator of the supercharges decomposes as follows:

$$
\left\{Q_{\alpha}, \bar{Q}_{\beta}\right\}=\Gamma_{\alpha \beta}^{M} P_{M}+\frac{1}{2} \Gamma_{\alpha \beta}^{M N} Z_{M N}+\frac{1}{5 !} \Gamma_{\alpha \beta}^{M N P Q R} Z_{M N P Q R} .
$$

Here the $P_{M}$ denote the 11-dimensional momentum operators and $Z_{M N}$ and $Z_{M N P Q R}$ are the charges associated with 2- and 5-branes. $P_{M}, Z_{M N}$ and $Z_{M N P Q R}$ represent $11+55+462=528$ components and thus generally parametrize the anticommutator on the right-hand side. Upon compactification on a torus $T^{n}$, where $n=11-d$, we are dealing with an extended supersymmetry algebra in $d$ spacetime dimensions. In this way one obtains corresponding centrally extended maximal supersymmetry algebras in lower dimensions. A priori these central charges transform according to representations of an internal $S O(n)$, but, in fact, there is a bigger group to which they can be assigned, namely to the automorphism group $H_{\mathrm{R}}$ of the supersymmetry algebra that acts on the supercharges and commutes with the $d$ dimensional Lorentz group (for a classification, see, e.g., [9]). It turns out, however, that this assignment can be further extended (although not in general as we shall discuss below), namely to representations of the hidden symmetry group $G$. This is shown in table 1 (see [10] for a comprehensive review).

According to (1) the pointlike central charges in $d$ spacetime dimensions can be classified, respectively, into 'Kaluza-Klein central charges' $P_{m}$ originating from the $D=11$ momentum operator $P_{M}$, and 'winding central charges' $Z_{m n}$ and $Z_{m n p q r}$ originating from $Z_{M N}$ and $Z_{M N P Q R}$. In this way we obtain (the number of central charge components is given in brackets)

$$
\begin{aligned}
d=9 \rightarrow P_{m}[2], & & Z_{m n}[1] \\
d=8 \rightarrow P_{m}[3], & & Z_{m n}[3] \\
d=7 \rightarrow P_{m}[4], & & Z_{m n}[6] \\
d=6 \rightarrow P_{m}[5], & & Z_{m n}[10] \oplus Z_{m n p q r}[1] \\
d=5 \rightarrow P_{m}[6], & & Z_{m n}[15] \oplus Z_{m n p q r}[6] \oplus Z_{\mu \nu \rho \sigma \tau}[1] \\
d=4 \rightarrow P_{m}[7], & & Z_{m n}[21] \oplus Z_{m n p q r}[21] \oplus Z_{m \mu \nu \rho \sigma}[7] \\
d=3 \rightarrow P_{m}[8], & & Z_{m n}[28] \oplus Z_{m n p q r}[56] \oplus Z_{m n \mu \nu \rho}[28] \\
d=2 \rightarrow P_{m}[9], & & Z_{m n}[36] \oplus Z_{\mu \nu}[1] \oplus Z_{m n p q r}[126] \oplus Z_{m n p \mu \nu}[84]
\end{aligned}
$$


Table 2. String-like central charges and their representations in dimensions $d \geqslant 5$. We indicate the branching into $H_{\mathrm{R}}$ representations for $d=6$.

\begin{tabular}{lll}
\hline$d$ & $G$ & Representations \\
\hline 9 & $S L(2, \mathbb{R}) \times S O(1,1)$ & $\mathbf{2}$ \\
8 & $S L(3, \mathbb{R}) \times S L(2, \mathbb{R})$ & $(\mathbf{3}, \mathbf{2})$ \\
7 & $S L(5, \mathbb{R})$ & $\mathbf{5}$ \\
6 & $S O(5,5)$ & $\mathbf{1 0} \oplus \mathbf{1} \rightarrow(\mathbf{5}, \mathbf{1}) \oplus(\mathbf{1}, \mathbf{5}) \oplus(\mathbf{1}, \mathbf{1})$ \\
5 & $E_{6(6)}$ & $\overline{\mathbf{2 7}}$ \\
\hline
\end{tabular}

where we used spacetime indices $\mu, v, \ldots=0, \ldots, d-1$ and internal indices $m, n, \ldots=$ $1, \ldots, 11-d$. These central charges transform according to representations of the group $H_{\mathrm{R}}$. In most cases this representation is irreducible (exceptions occur for $d=2,5$ and 9).

As indicated above, for $d \geqslant 4$ the central charges combine into representations of the bigger (hidden) symmetry groups $E_{n(n)}$. We already listed these representations in table 1 . Below $d=4$ the pointlike central charges no longer fit into representations of $G$, but only into representations of the $H_{\mathrm{R}}$ : for $d=3$, we obtain the $\mathbf{1 2 0}$ of $S O(16)$ (rather than a representation of $E_{8(8)}$ ), and for $d=2$ we have $\mathbf{1} \oplus \mathbf{1 2 0} \oplus \mathbf{1 3 5}$ of $S O(16)$ (rather than a representation of $\left.E_{9(9)}\right)$. In the latter case, the centrally extended maximal superalgebra in $d=2$ is given by

$$
\left\{Q_{ \pm}^{I}, Q_{ \pm}^{J}\right\}=\delta^{I J} P_{ \pm}, \quad\left\{Q_{+}^{I}, Q_{-}^{J}\right\}=Z^{I J},
$$

in terms of (one-component) Majorana-Weyl spinors with indices $I, J=1, \ldots, 16$. While left- or right-moving BPS states corresponding to elimination of either $Q_{+}^{I}$ or $Q_{-}^{I}$ (i.e. $(16,0)$ or $(0,16)$ supersymmetry) are massless and do not involve central charges, massive states involving the 256 central charges $Z^{I J}$ have not been considered in the literature so far.

Similar considerations apply to the central charges with Lorentz indices, such as the "stringlike' central charges with one spacetime index; these charges are carried by one-dimensional extended objects (strings) rather than point particles. We obtain

$$
\begin{aligned}
& d=9 \rightarrow Z_{\mu m}[2] \\
& d=8 \rightarrow Z_{\mu m}[3] \\
& d=7 \rightarrow Z_{\mu m}[4] \oplus Z_{\mu m n p q}[1] \\
& d=6 \rightarrow Z_{\mu m}[5] \oplus Z_{\mu m n p q}[5] \oplus Z_{\mu v \rho \sigma \tau}[1] \\
& d=5 \rightarrow Z_{\mu m}[6] \oplus Z_{\mu m n p q}[15] \oplus Z_{\mu v \rho \sigma m}[6] \\
& d=4 \rightarrow Z_{\mu m}[7] \oplus Z_{\mu m n p q}[35] \oplus Z_{\mu v \rho m n}[21] \\
& d=3 \rightarrow Z_{\mu \nu}[1] \oplus Z_{\mu m}[8] \oplus Z_{\mu m n p q}[70] \oplus Z_{\mu \nu m n p}[56] \\
& d=2 \rightarrow Z_{\mu m}[9] \oplus Z_{\mu m n p q}[126] .
\end{aligned}
$$

Again these charges transform according to the group $H_{\mathrm{R}}$. For $d \geqslant 5$ they also fit into representations of $G$; these cases are listed in table 2. For $d=4$ we have a 63 of $S U(8)$ (and 
not a representation of $E_{7(7)}$ ). For $d=3,2$ we have a 135 representation of $S O(16)$ (and not a representation of $E_{8(8)}$ or $E_{9(9)}$, respectively). The same pattern is seen for the 2-brane charges, which only fit into representations of the hidden symmetry group for $d \geqslant 6$. For higher brane charges the phenomenon does not occur, perhaps because the symmetry groups become simpler and so do the representations. It is noteworthy that the failure of the pointlike, string-like and 2-brane charges to constitute representations of $G$ takes place at those dimensions where the corresponding gauge fields (with rank 1, 2 and 3, respectively) can be dualized to scalars or where they cease to exist altogether.

It is evident that the toroidal compactifications of maximal supergravity are incomplete, even when retaining the Kaluza-Klein states. While correctly describing the dynamics of the massless sectors they do not incorporate the full set of BPS states, simply because the charges $Z_{M N}$ and $Z_{M N P Q R}$ remain zero. It is obvious that some of these missing charges can be generated by solitonic solutions (or they can be introduced explicitly into the field theory). Nevertheless, there seem to remain certain deficiencies in the spectrum. For example, there is a 3-brane central charge transforming according to $(\mathbf{1 0}, \mathbf{1}) \oplus(\mathbf{1}, \mathbf{1 0})$ of $U S p(4) \times U S p(4)$ in $d=6$. However, there are no massless fields and certainly no 4-rank gauge fields present in this representation. So it seems rather difficult to envisage solutions with the required central charge. Furthermore, solitonic solutions are expected to transform into other solutions under $G$, which represents after all a symmetry of the equations of motion. Thus one would expect the central charge lattice to eventually cover all possible values consistent with the group $G$, and not just with $H_{\mathrm{R}}$. However, the supersymmetry algebra itself does not allow for all central charges consistent with the group $G$.

Let us recall that the hidden symmetries of the toroidal compactifications of $d=11$ supergravity [1] are realized as continuous symmetries upon truncation to the massless modes. When including the BPS modes, the symmetry can no longer be realized in this form. The central charge values will constitute a certain lattice and the hidden symmetry group will be restricted to an arithmetic subgroup of $E_{n(n)}$ that leaves the charge lattice invariant. It has been conjectured [2] that this arithmetic subgroup, called $U$-duality, is, in fact, a symmetry group of the full M-theory (compactified on a hyper-torus). The fact that, in low dimensions, the central charges cannot be assigned to representations of the hidden symmetry casts some doubt on the assertion that $E_{8(8)}(Z)$ and $E_{9(9)}(Z)$ are symmetries of M-theory reduced to three and two dimensions, respectively. Similar comments apply to the relevance of $E_{7(7)}(Z)$ and $E_{6(6)}(Z)$ for the string-like and membrane-like solitonic excitations in $d=4$ and 5 , respectively. In fact, below three dimensions the situation is even less clear, because the canonical realization of the hidden global symmetry does not yield the expected affine algebra $E_{9(9)}$, but rather a quadratic (Yangian) algebra over $E_{8(8)}[11]$.

Another question that poses itself is that, if M-theory does indeed possess the $U$-dualities as a symmetry of the full theory, then some trace of the $E_{n(n)}$ symmetry of the compactified theory could still be present at the level of $d=11$ supergravity. This expectation is not unreasonable in view of the fact that, while the towers of Kaluza-Klein states are not in representations of $E_{n(n)}$, one could envisage completing the theory by adding some of the missing BPS states in order to regain some (approximate) invariance. Hence it is of interest to see whether $d=11$ supergravity, without truncation to the massless states in some toroidal compactification, has any features reminiscent of the $E_{n(n)}$ symmetry that one finds in the truncated theories. As we will argue in the next section, this indeed turns out to be the case. In section 4 we will then discuss the possibilities for modifying $d=11$ supergravity in such a way that we regain (some part of) the $E_{n(n)}$ invariances. 


\section{Hidden symmetries in 11 dimensions}

Our discussion of central charges and the way in which they arise from the $d=11$ ancestor theory raises the question as to what the role of the hidden $E_{n(n)}$ symmetries is in the context of that theory. As we mentioned already in the previous section it has been conjectured that an arithmetic subgroup of the nonlinearly realized $E_{n(n)}$ symmetries is an exact symmetry of (toroidally compactified) M-theory. On the other hand, $d=11$ supergravity as such only exhibits this invariance when truncated to the massless fields in a toroidal compactification. From the perspective of that compactification the massive Kaluza-Klein states are responsible for the fact that the invariance is lost for $d=11$ supergravity. This is not surprising in view of the fact that, as noted in the previous section, the Kaluza-Klein states are incomplete and do not constitute $E_{n(n)}$ multiplets of BPS states. This observation suggests that it may be possible to extend the theory in such a way that an arithmetic subgroup of $E_{n(n)}$ could become an exact invariance. We will turn to this question in the next section, but here we wish to point out that, indeed, full uncompactified $d=11$ supergravity shows traces of the hidden symmetries, which suggests that they appear not merely in special compactifications and corresponding truncations. We review the evidence for this idea which was presented in $[6,7]$ already some time ago, and discuss it in the light of the more recent developments. There are intriguing indications of a hidden 'exceptional geometry' of $d=11$ supergravity that remains to be discovered (see [12] for a more recent discussion of this point). While the work of [6] was aimed chiefly at establishing the consistency of the Kaluza-Klein truncation of $d=11$ supergravity compactified on $S^{7}$ to its massless sector [18], we are thus motivated here by the desire to understand the dynamics of the nonperturbative M-theory degrees of freedom, and the role of hidden symmetries in the full Mtheory.

Let us first summarize the main results of $[6,7]$, where alternative versions of $d=11$ supergravity were constructed with local $S O(1,3) \times S U(8)$ and $S O(1,2) \times S O(16)$ tangentspace symmetries, respectively, which are gauge equivalent to the original version of [1]. This equivalence holds at the level of the equations of motion ${ }^{3}$. In both of these new versions the supersymmetry variations acquire a polynomial form from which the corresponding formulae for the maximal supergravities in four and three dimensions can be read off directly and without the need for any duality redefinitions. This reformulation can thus be regarded as a step towards the complete fusion of the bosonic degrees of freedom of $d=11$ supergravity (i.e. the elfbein $E_{M}{ }^{A}$ and the antisymmetric tensor $A_{M N P}$ ) in a way which is in harmony with the hidden symmetries of the dimensionally reduced theories.

For lack of space we restrict attention to the bosonic sector, and first describe the version of [6] exposing a hidden $E_{7(7)}$ structure of $d=11$ supergravity. There is also a version involving $E_{8(8)}$ [7], but in order not to overburden the notation with too many different kinds of indices, we will summarize the pertinent results separately in the second part of this section. At any rate, readers are advised to consult the original papers for further explanations and the more technical details of the construction.

The first step in the procedure is to break the original tangent-space symmetry $S O(1,10)$ into its subgroups $S O(1,3) \times S O(7)$ and $S O(1,2) \times S O(8)$, respectively, through a partial choice of gauge for the elfbein. In a second step, one enlarges these symmetries again to $S O(1,3) \times S U(8)$ and $S O(1,2) \times S O(16)$ by introducing new gauge degrees of freedom. This symmetry enhancement requires suitable redefinitions of the bosonic and fermionic fields, and their combination into tensors with respect to the new tangent-space symmetries.

3 Recall that even the Lagrangian of $N=8$ supergravity [5] cannot be obtained directly from 11 dimensions; to exhibit the hidden symmetries, one must pass through the equations of motion. 
The construction thus requires $4+7$ and $3+8$ splits of the $d=11$ coordinates and indices, respectively, implying a similar split for all tensors of the theory. It is important, however, that the dependence on all 11 coordinates is retained throughout. The alternative theory remains fully equivalent to the original formulation of [1] upon suitable gauge choices.

The elfbein and the three-index photon are thus combined into new objects covariant with respect to the new tangent-space symmetry. In the special Lorentz gauge preserving either $S O(1,3) \times S O(7)$ or $S O(1,2) \times S O(8)$ the elfbein takes the form

$$
E_{M}{ }^{A}=\left(\begin{array}{cc}
\Delta^{-s} e_{\mu}{ }^{a} & B_{\mu}{ }^{m} e_{m}{ }^{a} \\
0 & e_{m}{ }^{a}
\end{array}\right),
$$

where curved $d=11$ indices are decomposed as $M=(\mu, m)$ with $\mu=0,1,2(, 3)$ and $m=(3) 4,, \ldots, 10$, with a similar decomposition of the flat indices; furthermore, $\Delta:=\operatorname{det} e_{m}{ }^{a}$ and $s=1 /(d-2)$ for $d=4$ and 3, respectively. In this gauge, the elfbein contains the (Weyl rescaled) drei- or vierbein and the Kaluza-Klein vectors $B_{\mu}{ }^{m}$. The internal vielbein is replaced by a new object, which we refer to as a generalized vielbein, and which can be identified by a careful analysis of the supersymmetry variation of $B_{\mu}{ }^{m}$. For the $4+$ 7 split, this generalized vielbein, denoted by $e_{A B}^{m}$, carries an upper internal world index, and has lower $S U(8)$ indices $A, B=1, \ldots, 8$. It is antisymmetric in the indices $A, B$ so that it transforms into the $\mathbf{2 8}$ representation. Explicitly,

$$
e_{A B}^{m} \rightarrow U_{A}^{C} U_{B}^{D} e_{C D}^{m}
$$

where $U_{A}{ }^{C}$ is an $S U(8)$ matrix depending on all 11 coordinates. By including its complex conjugate,

$$
e^{m A B}:=\left(e_{A B}^{m}\right)^{*},
$$

the generalized vielbein is thus given by the complex tensor $\left(e^{m A B}, e_{A B}^{m}\right)$, which, for given $m$, constitutes the 56 (pseudo-real) representation of $E_{7(7)}$; according to its maximal compact subgroup $S U(8)$ this representation branches into $\mathbf{2 8} \oplus \overline{\mathbf{2 8}}$

The generalized vielbein contains the original siebenbein $e_{m}{ }^{a}$, as can be seen by choosing a special $S U(8)$ gauge such that

$$
e_{A B}^{m}:=\mathrm{i} \Delta^{-1 / 2} e_{a}^{m} \Gamma_{A B}^{a},
$$

where $m=4, \ldots, 10$ and $\Gamma^{a}$ are the standard $S O(7) \Gamma$-matrices (our conventions are such that $e_{A B}^{m}$ is real in this gauge). Being the inverse densitized internal siebenbein contracted with an $S O$ (7) $\Gamma$-matrix, our generalized vielbein object is very much analogous to the inverse densitized triad in Ashtekar's reformulation of Einstein's theory [14].

The generalized vielbein has many more components than the original siebenbein, but of course the number of physical degrees of freedom is the same as before. Some of the redundant degrees of freedom are taken care of by the $S U(8)$ gauge symmetry, but further algebraic constraints must exist to match the original physical content of the theory. These constraints are indeed present and can be derived by making use of properties of $S O(7) \Gamma$ matrices. An obvious one is the 'Clifford property', already identified in [6]:

$$
e_{A C}^{m} e^{n C B}+e_{A C}^{n} e^{m C B}=\frac{1}{4} \delta_{A}^{B} e_{C D}^{m} e^{n D C} .
$$


Furthermore, from (8) one obtains a formula for the original 7-metric,

$$
(\operatorname{det} g)^{-s} g^{m n}=\frac{1}{8} e_{C D}^{m} e^{n C D},
$$

in terms of the new generalized vielbein, which immediately yields the 'master formula' for the full nonlinear metric ansatz in the Kaluzu-Klein reduction of $d=11$ supergravity. This formula has been exploited in recent work on the AdS/CFT correspondence (see, e.g., [13]).

The Clifford property is by itself not enough to reduce the number of physical degrees of freedom to the desired one. Rather, it is part of the following set of $E_{7(7)}$ covariant constraints:

$$
\begin{aligned}
& e_{A B}^{m} e^{n A B}-e_{A B}^{n} e^{m A B}=0, \\
& e_{A C}^{m} e^{n C B}+e_{A C}^{n} e^{m C B}-\frac{1}{4} \delta_{A}^{B} e_{C D}^{m} e^{n D C}=0, \\
& e_{[A B}^{m} e_{C D]}^{n}-\frac{1}{24} \varepsilon_{A B C D E F G H} e^{m E F} e^{n G H}=0 .
\end{aligned}
$$

These equations correspond to the singlet and the $\mathbf{1 3 3}$ in the $E_{7(7)}$ decomposition,

$$
56 \otimes 56 \rightarrow 1 \oplus 133 \oplus 1463 \oplus 1539 .
$$

The constraints can thus be rephrased as the statement that the product $e^{m} \otimes e^{n}$ only contains the 1463 and 1539 representations of $E_{7(7)}$.

In addition to the algebraic constraints, the generalized vielbein satisfies a set of differential relations, called the 'generalized vielbein postulate' in [6]. In order to state them, we need suitable $E_{7(7)}$ connections $Q_{M}{ }^{A}{ }_{B}$ and $P_{M}^{A B C D}=\frac{1}{24} \varepsilon^{A B C D E F G H} P_{M E F G H}$ in 11 dimensions. These are built out of the $S O(1,10)$ coefficients of anholonomity and the four-index field strength $F_{M N P Q}$ of $d=11$ supergravity in the way explained in [6]; since the explicit expressions are somewhat cumbersome we refer readers there for details. The vector $Q_{M}{ }_{B}{ }_{B}$ acts as the connection for the local $S U(8)$ transformations and is therefore in the 63 representation of that group. The tensor $P_{M}^{A B C D}$ transforms as the self-dual $\mathbf{3 5}$ under the action of $S U(8)$. Together they constitute the (adjoint) 133 representation of $E_{7(7)}$. For the massless theory these quantities are directly related to the pull-backs to $d=11$ spacetime of the tangent-space connection and vielbein associated with the homogeneous space $E_{7(7)} / S U(8)$.

The generalized vielbein postulate takes the form

$\mathcal{D}_{\mu} e_{A B}^{m}+\frac{1}{2} \mathcal{D}_{n} B_{\mu}{ }^{n} e_{A B}^{m}+\mathcal{D}_{n} B_{\mu}{ }^{m} e_{A B}^{n}+2 Q_{\mu[A}{ }^{C} e_{B] C}^{m}+P_{\mu A B C D} e^{m C D}=0$,

$\mathcal{D}_{n} e_{A B}^{m}+2 Q_{n[A}{ }^{C} e_{B] C}^{m}+P_{n A B C D} e^{m C D}=0$,

where

$$
\mathcal{D}_{\mu}:=\partial_{\mu}-B_{\mu}{ }^{m} \mathcal{D}_{m}
$$

for $\mu=0,1,2,3$ and

$$
\begin{aligned}
& \mathcal{D}_{m} e_{A B}^{n}:=\partial_{m} e_{A B}^{n}+\Gamma_{m p}{ }^{n} e_{A B}^{p}+\frac{1}{2} \Gamma_{m p}{ }^{p} e_{A B}^{n}, \\
& \mathcal{D}_{m} B_{\mu}{ }^{n}:=\partial_{m} B_{\mu}{ }^{n}+\Gamma_{m p}{ }^{n} B_{\mu}{ }^{p},
\end{aligned}
$$

for the internal indices. The extra term with $\Gamma_{m p}{ }^{p}$ in the above relation arises because the generalized vielbein transforms as a density. Observe that the affine connection $\Gamma_{m n}{ }^{p}$ still depends on all 11 coordinates, and that the covariance of these relations under general internal coordinate transformations (with parameters $\xi^{m}(x, y)$ ) had not been previously exhibited in [6] 
where the generalized vielbein postulate was given without the affine connections. The extra terms are obtained by uniformly replacing

$$
e_{a}{ }^{n} \partial_{m} e_{n b} \rightarrow e_{a}^{n}\left(\partial_{m} e_{n b}-\Gamma_{m n}^{p} e_{p b}\right),
$$

in the relevant formulae of $[6,7]$ defining the connection. For the explicit verification of the above relations with the fully covariant derivatives, it is furthermore useful to observe that the relevant terms appearing in the connection coefficients $Q_{\mu}$ and $P_{\mu}$ contain the combination

$$
e_{a}{ }^{m}\left(\partial_{m} B_{\mu}{ }^{n} e_{n b}+B_{\mu}{ }^{n} \partial_{n} e_{m b}\right)=e_{a}{ }^{m}\left(\partial_{m}\left(B_{\mu}{ }^{n} e_{n b}\right)+2 B_{\mu}{ }^{n} \partial_{[n} e_{m] b}\right),
$$

which remains unchanged if we replace $\partial_{m}$ by $\mathcal{D}_{m}$, provided the affine connection is torsionfree.

We emphasize that the affine connection is still arbitrary at this point, as it cancels between the different terms in the generalized vielbein postulate. A convenient choice is the standard Christoffel connection, which is obtained by setting

$$
\mathcal{D}_{m}\left(\Delta^{-1} g^{n p}\right)=2 P_{m}^{A B C D} e_{A B}^{n} e_{C D}^{p}=0 .
$$

So we conclude that all the quantities introduced above comprise $E_{7(7)}$ representations. We stress once again that we are still dealing with the full $d=11$ supergravity theory. This pattern continues. For instance, the supersymmetry variation of the generalized vielbein takes a form that closely resembles the four-dimensional transformation rule for the massless modes (in the truncation to the massless modes, $e_{A B}^{m}$ is proportional to the $E_{7(7)} / S U(8)$ coset representative),

$$
\delta e_{A B}^{m}=-\sqrt{2} \Sigma_{A B C D} e^{m C D},
$$

where

$$
\Sigma_{A B C D}=\bar{\epsilon}_{[A} \chi_{B C D]}+\frac{1}{24} \epsilon^{E} \chi^{F G H},
$$

where $\epsilon_{A}$ and $\chi_{A B C}$ denote the supersymmetry parameters and the spin- $\frac{1}{2}$ fields, respectively. It takes a little more work to check that the covariantizations with respect to $\Gamma_{m n}{ }^{p}$ introduced above do not alter the form of the supersymmetry variations given in [6], except for extra terms involving $\Gamma_{m n}{ }^{n}$ necessary because the redefined supersymmetry transformation parameter is also a density of weight $\frac{1}{4}$ (the original supersymmetry parameter is of zero weight). Similarly, the bosonic and fermionic equations of motion can be cast into a fully $S U(8)$ covariant form.

In spite of the fact that the theory can be formulated elegantly in terms of $E_{7(7)}$ quantities, it cannot be invariant under $E_{7(7)}$. The obvious reason for that is the presence of the Kaluza-Klein gauge fields $B_{\mu}{ }^{m}$, which do not constitute a proper representation. However, when restricting ourselves to the massless modes in the toroidal representations, these fields disappear in the generalized vielbein postulate. On the other hand, the inability to preserve $E_{7(7)}$ invariance for these fields is precisely related to the deficiencies in the central charge assignments that we discussed in the previous section. If this deficiency could somehow be lifted, then it might be possible to regain the hidden symmetry with BPS states present.

For the $3+8$ split one has analogous results $[7,12,15]$, but with a different decomposition of indices. The hidden symmetry of the theory is $E_{8(8)}[16,17]$ with a local $S O(16)$ replacing the $S U(8)$ of the $4+7$ split. The $S O(16)$ vector representation 16 is labelled by indices $I, J=1, \ldots, 16$, while $A, B=1, \ldots, 128$ labels the $\mathbf{1 2 8}_{s}$ chiral spinor and $\dot{A}, \dot{B}=1, \ldots, 128$ the $\mathbf{1 2 8}_{c}$ opposite-chirality spinor of $S O(16)$. The $S O(8)$ tangent space 
group of the original $d=11$ theory is embedded into $S O(16)$ according to $\mathbf{1 6}_{v} \rightarrow \mathbf{8}_{s} \oplus \mathbf{8}_{c}$. The two spinor representations then branch according to $\mathbf{1 2 8}_{s} \rightarrow\left(\mathbf{8}_{v} \otimes \mathbf{8}_{v}\right) \oplus\left(\mathbf{8}_{s} \otimes \mathbf{8}_{c}\right)$, and according to $\mathbf{1 2 8}_{c} \rightarrow\left(\mathbf{8}_{v} \otimes \mathbf{8}_{c}\right) \oplus\left(\mathbf{8}_{s} \otimes \mathbf{8}_{v}\right)$. The $S O(16)$ adjoint representation 120 and the spinor representation $\mathbf{1 2 8}$ (or its adjoint) constitute the $\mathbf{2 4 8}$ representation of $E_{8(8)}$, where we remind the reader of the well known fact (relevant below) that the adjoint and the fundamental representation of this group coincide. Hence the $\mathbf{2 4 8}$ representation can be labelled by the indices $\mathcal{A}=([I J], A)$ according to the $S O(16)$ decomposition $\mathbf{1 2 0} \oplus \mathbf{1 2 8}$. Observe that the $S O(16)$ index pairs $[I J]$ correspond to the $S O(8)$ index pairs $[\alpha \beta], \alpha \dot{\beta}$ and $[\dot{\alpha} \dot{\beta}]$; the $S O(16)$ spinor indices $A$ and $\dot{A}$ also correspond to $S O(8)$ index pairs, namely to $a b$ and $\alpha \dot{\beta}$ and to $a \alpha$ and $a \dot{\alpha}$, respectively. Here the $S O(8)$ indices $a, \alpha, \dot{\alpha}$ label the $\mathbf{8}_{v}, \mathbf{8}_{s}$ and $\mathbf{8}_{c}$ representations of $S O(8)$, respectively.

The matter-like bosonic degrees of freedom are now combined into a generalized vielbein $e_{\mathcal{A}}^{m} \equiv\left(e_{I J}^{m}, e_{A}^{m}\right)$ on which local $S O(16)$ acts reducibly according to

$$
e_{\mathcal{A}}^{m} \rightarrow U_{\mathcal{A}}^{\mathcal{B}} e_{\mathcal{B}}^{m}
$$

with $U_{\mathcal{A}}{ }^{\mathcal{B}}$ in the $120 \oplus \mathbf{1 2 8}$ representation. As before, we can relate this new vielbein to the original achtbein in a special gauge. In order to avoid introducing yet more notation we refer readers to [7] for details, and simply quote the result,

$$
\left(e_{I J}^{m}, e_{A}^{m}\right):= \begin{cases}\Delta^{-1} e_{a}{ }^{m} \Gamma_{\alpha \dot{\beta}}^{a} & \text { if }[I J] \text { or } A=\alpha \dot{\beta}, \\ 0 & \text { otherwise. }\end{cases}
$$

As before, the $E_{8(8)}$ vielbein $e_{\mathcal{A}}^{m}$ has far more components than there are physical degrees of freedom, and therefore must again satisfy a number of algebraic constraints [15]. We have

$$
e_{A}^{m} e_{A}^{n}-\frac{1}{2} e_{I J}^{m} e_{I J}^{n}=0,
$$

and

$$
\Gamma_{A B}^{I J}\left(e_{B}^{m} e_{I J}^{n}-e_{B}^{n} e_{I J}^{m}\right)=0, \quad \Gamma_{A B}^{I J} e_{A}^{m} e_{B}^{n}+4 e_{K[I}^{m} e_{J] K}^{n}=0
$$

where $\Gamma_{A \dot{A}}^{I}$ are the standard $S O(16) \Gamma$-matrices and $\Gamma_{A B}^{I J} \equiv\left(\Gamma^{[I} \Gamma^{J]}\right)_{A B}$; the minus sign in (22) reflects the fact that we are dealing with the maximally non-compact $E_{8(8)}$. Obviously, (22) and (23) correspond to the singlet and the adjoint representations of $E_{8(8)}$ in the product $e^{m} \otimes e^{n}$. More complicated are the following relations transforming in the $\mathbf{3 8 7 5}$ representation of $E_{8(8)}$ :

$$
\begin{aligned}
& e_{I K}^{(m} e_{J K}^{n)}-\frac{1}{16} \delta_{I J} e_{K L}^{m} e_{K L}^{n}=0, \\
& \Gamma_{\dot{A} B}^{K} e_{B}^{(m} e_{I K}^{n)}-\frac{1}{14} \Gamma_{\dot{A} B}^{I K L} e_{B}^{(m} e_{K L}^{n)}=0, \\
& e_{[I J}^{(m} e_{K L]}^{n)}+\frac{1}{24} e_{A}^{m} \Gamma_{A B}^{I J K L} e_{B}^{n}=0 .
\end{aligned}
$$

These relations can be elegantly summarized by means of $E_{8(8)}$ projectors [12]

$$
\left(\mathcal{P}_{\mathrm{j}}\right)_{\mathcal{A B}}{ }^{\mathcal{C D}} e^{m} e^{n}{ }_{\mathcal{D}}^{n}=0
$$

for the $j=\mathbf{1}, \mathbf{2 4 8}$ and $\mathbf{3 8 7 5}$ representations appearing in the product,

$$
248 \otimes 248 \rightarrow 1 \oplus \mathbf{2 4 8} \oplus \mathbf{3 8 7 5} \oplus \mathbf{2 7 0 0 0} \oplus \mathbf{3 0 3 8 0} .
$$

In comparison with the $4+7$ case we note the appearance of a fifth representation in this decomposition corresponding to $\mathbf{3 8 7 5}$ which has no analogue in $E_{7(7)}$. 
For the differential relations we again need composite connections that belong to the Lie algebra of $E_{8(8)}$. These have components

$$
\mathcal{Q}_{M}{ }^{\mathcal{A}} \equiv\left(Q_{M}{ }^{I J}, P_{M}{ }^{A}\right),
$$

whose explicit expressions in terms of the $d=11$ coefficients of anholonomity and the fourindex field strength $F_{M N P Q}$ can be found in [7]. The generalized vielbein postulate now takes an even simpler form: with the $E_{8(8)}$ structure constants $f_{\mathcal{A B}}{ }^{\mathcal{C}}$, we have

$$
\begin{aligned}
& \mathcal{D}_{\mu} e^{m}{ }_{\mathcal{A}}+\mathcal{D}_{n} B_{\mu}{ }^{n} e^{m}{ }_{\mathcal{A}}+\mathcal{D}_{n} B_{\mu}{ }^{m} e^{n}{ }_{\mathcal{A}}+f_{\mathcal{A B}}{ }^{\mathcal{C}} \mathcal{Q}_{\mu}{ }^{\mathcal{B}} e^{m}{ }_{\mathcal{C}}=0, \\
& \mathcal{D}_{n} e^{m}{ }_{\mathcal{A}}+f_{\mathcal{A B}}{ }^{\mathcal{C}} \mathcal{Q}_{n}{ }^{\mathcal{B}} e^{m}{ }_{\mathcal{C}}=0,
\end{aligned}
$$

where $\mathcal{D}_{\mu}$ and $\mathcal{D}_{m}$ are the same as before, except for the fact that the generalized vielbein transforms as a density of different weight compared with the case $d=4$ (cf (8) and (21)). Like (22)-(24), the differential relations are thus covariant under general coordinate transformations as well as $E_{8(8)}$. Just as before, we note that the full theory does not respect $E_{8(8)}$ invariance.

The new versions yield the maximal supergravities in $d=4$ and 3 directly and without further ado. In particular, one obtains in this way the $S U(8)$ and $S O(16)$ covariant equations of motion, which combine equations of motion and Bianchi identities of the original theory in 11 dimensions [6]. Furthermore, the reduction of $d=11$ supergravity to three dimensions yields $d=3, N=16$ supergravity [17], and is accomplished rather easily, since no duality redefinitions are needed any more, unlike in [5]. The propagating bosonic degrees of freedom in three dimensions are all scalar, and combine into a matrix $\mathcal{V}(x)$, which is an element of a non-compact $E_{8(8)} / S O(16)$ coset space, and whose dynamics is governed by a nonlinear $\sigma$-model coupled to $d=3$ gravity. The identification of the 248-bein with the $\sigma$-model field $\mathcal{V} \in E_{8(8)}$ is given by

$e_{I J}^{m}(x)=\frac{1}{60} \operatorname{Tr}\left[Z^{m} \mathcal{V}(x) X^{I J} \mathcal{V}^{-1}(x)\right], \quad e_{A}^{m}(x)=\frac{1}{60} \operatorname{Tr}\left[Z^{m} \mathcal{V}(x) Y^{A} \mathcal{V}^{-1}(x)\right]$

where $X^{I J}$ and $Y^{A}$ are the compact and non-compact generators of $E_{8(8)}$, respectively, and where the $Z^{m}$ for $m=3, \ldots, 10$ are eight commuting nilpotent generators (hence obeying $\operatorname{Tr}\left(Z^{m} Z^{n}\right)=0$ for all $m$ and $\left.n\right)$. The verification of these assertions, and in particular of (24), relies on the very special properties of the $E_{8(8)}$ Lie algebra (we refer to [12] for details).

A very interesting aspect of the $3+8$ split is that the above relations can be exploited to argue that the $E_{8(8)}$ matrix $\mathcal{V}$ is present already in 11 dimensions and thus depends on the 11dimensional coordinates [12]. Namely, the fundamental and adjoint representations of $E_{8(8)}$ being the same, we have

$$
\mathcal{V}^{-1}(x, y) t^{\mathcal{A}} \mathcal{V}(x, y)=\mathcal{V}^{\mathcal{A}}{ }_{\mathcal{B}}(x, y) t^{\mathcal{B}}
$$

for all $E_{8(8)}$ generators $t^{\mathcal{A}}$. Hence,

$$
e^{m}{ }_{\mathcal{A}}(x, y)=\mathcal{V}^{m}{ }_{\mathcal{A}}(x, y) .
$$

Put differently, the generalized vielbein (as a rectangular matrix) is a submatrix of a full 248bein in 11 dimensions! This suggests that we should enlarge the range of indices $m$ to run over the whole group $E_{8(8)}$, with a corresponding increase in the number of dimensions, which, as we will see, is also suggested by the analysis of central charges. A first step in this direction was taken in [12], where it was shown that the 28 further vector fields originating from the 3 -form potential of $d=11$ supergravity necessitate the introduction of 28 further components $e_{m n \mathcal{A}}$, thereby enlarging the range of indices to 36 . 
Evidently many of the formulae displayed above are trivially satisfied for toroidal compactifications - for instance, all the connection components in the internal dimensions simply vanish in the truncation to the massless sector. One would therefore like to check the above results in the context of compactifications of $d=11$ supergravity on non-trivial internal manifolds, as such compactifications can provide valuable 'models' for the exceptional geometry that we have been alluding to. To date there is only one model of this type, namely the $A d S_{4} \times S^{7}$ compactification of $d=11$ supergravity [18] (the $A d S_{7} \times S^{4}$ truncation of [19] could eventually provide another model, but those results remain to be analysed from the point of view taken here). In that case, the internal connection components $Q_{m}{ }^{A}{ }_{B}$ and $P_{m}^{A B C D}$ do survive the truncation to the massless modes and are metamorphosed into the ' $T$ tensor' describing the couplings of the scalars and the fermions in gauged supergravity [18,20]. This is related to the remarkable fact that for gauged $N=8$ supergravity in four and five dimensions, this $T$-tensor actually transforms as a representation of the $E_{n(n)}$ symmetry group (for $n=7$ and $n=6$, respectively), even though $E_{n(n)}$ is no longer a symmetry of the gauged theory! More specifically, for $d=4$ we have [21]

$$
\mathbf{3 6} \oplus \mathbf{4 2 0}+\text { c.c. } \quad(\text { of } S U(8)) \longrightarrow \mathbf{9 1 2} \quad\left(\text { of } E_{7(7)}\right),
$$

while for $d=5$ [22]

$$
\left.36 \oplus 315 \quad \text { (of } U S p(8)) \longrightarrow 351 \quad \text { (of } E_{6(6)}\right) \text {. }
$$

The analogous decomposition for $d=3$, namely

$$
135 \oplus \mathbf{1 8 2 0} \oplus \mathbf{1 9 2 0} \quad(\text { of } S O(16)) \longrightarrow \mathbf{3 8 7 5} \quad \text { (of } E_{8(8)} \text { ), }
$$

has recently been invoked to construct a gauged maximal supergravity in three dimensions [23]. There, the consistency of the gauged theory is imposed by searching for gauge groups such that the $T$-tensor admits precisely the decomposition (34), thereby turning the derivation of [20] upside down.

\section{BPS-extended supergravity}

In the previous section we reviewed the evidence for hidden $E_{7(7)}$ and $E_{8(8)}$ structures of $d=11$ supergravity. However, the fact that certain sectors of the theory assume an $E_{n(n)}$ covariant form does not mean that $E_{n(n)}$ is actually a symmetry of $d=11$ supergravity, as is already obvious from the fact that the vector fields $B_{\mu}{ }^{m}$ in equation (14) cannot be assigned to representations of $E_{n(n)}$. Rather, our results should be interpreted as an indication that there exist extensions of $d=11$ supergravity which do possess these symmetries. Recent developments in string theory have led to the conjecture that the so-called $U$-duality group, which is an integer-valued subgroup of the nonlinearly realized $E_{n(n)}$ symmetries is actually an exact symmetry of (toroidally compactified) M-theory and therefore acts on the BPS states as well (see, e.g., [10] for a recent review). It is therefore not unreasonable to conjecture the existence of yet larger theories unifying the BPS degrees of freedom. We will refer to the effective field theories incorporating all these degrees of freedom as 'BPS extended supergravities'.

The existence of central charges other than those associated with the momentum states on the hyper-torus strongly suggests that there are extra spacetime dimensions that would be similarly associated with the remaining central charges. Such an extension would, in particular, imply the existence of further Kaluza-Klein vector fields in (14), which would then couple to the non-momentum central charges. In most compactifications one has precisely 
the right number of vector gauge fields. In five dimensions there is one singlet pointlike central charge without a corresponding gauge field; in four dimensions there are 28 gauge fields and 56 central charges. The extra 28 charges are associated with monopoles and here the charges are mutually non-local in the sense that one cannot incorporate electric and magnetic charges simultaneously in a local field theory. Admittedly this may be an obstacle in associating extra dimensions to all the 56 central charges. The idea of introducing extra dimensions for the description of supersymmetric theories with central charges is, in fact, an old one [24], but we believe that the results reviewed above make it even more compelling. We would therefore expect there to be bigger theories existing in $4+56$ dimensions (for the $E_{7(7)}$ version of [6]), and $3+248$ dimensions (for the $E_{8(8)}$ version of [7]). However, it remains to be seen whether these extra dimensions are really on the same footing as ordinary spacetime dimensions. In this section, we retreat a little in order to explore the implications of this idea in the context of a simpler example, namely $d=9$ supergravity, where there are only three central charges (cf table 1). In that case, one has not only a detailed understanding of certain BPS states, contained in so-called KKA and KKB supermultiplets [25], but there is also a candidate theory whose effective field theory description contains both the supergravity and the BPS degrees of freedom, as well as the massive IIA and IIB superstring degrees of freedom, and would therefore serve as a prime example of the BPS-extended supergravity that we have in mind here, namely the supermembrane [26]! This point was, in fact, already made in [27] where it was first proposed to view the Kaluza-Klein and the winding states on the M-theory torus as manifestations of an underlying supermembrane theory. In dimensions less than nine, yet more (pointlike) BPS degrees of freedom will arise. This suggests the existence of yet bigger theories 'beyond the supermembrane' which eventually would also account for the states associated with the 5-brane charges.

The compactification of M-theory on a torus $T^{2}$ is expected to comprise both IIA and IIB superstring theory, and is hence conjectured to be invariant under the $S$-duality group $S L(2, Z)$. In 10 spacetime dimensions the massive supermultiplets of IIA and IIB string theory coincide, whereas the massless states comprise inequivalent supermultiplets, for the simple reason that they transform according to different representations of the $S O(8)$ helicity group. When compactifying the theory on a circle, massless states IIA and IIB states in nine spacetime dimensions transform according to identical $S O(7)$ representations of the helicity group and constitute equivalent supermultiplets. The corresponding interacting field theory is the unique $N=2$ supergravity theory in nine spacetime dimensions. However, the supermultiplets of the BPS states, which carry momentum along the circle, remain inequivalent, as they remain assigned to the inequivalent representations of the group $S O(8)$ which is now associated with the rest frame (spin) rotations of the massive states. The Kaluza-Klein states of the IIA theory constitute the so-called KKA supermultiplets, whereas those of the IIB theory constitute the (inequivalent) KKB multiplets. It was proven in [25] that the IIA winding states constitute KKB supermultiplets and the IIB winding states constitute KKA supermultiplets, so that $T$-duality remains valid.

One can obtain the same result for the 11-dimensional (super)membrane, which contains excitations corresponding to all the BPS states found in the supermultiplet analysis. Assuming that the 2-brane charge takes values only in the compact coordinates labelled by 9 and 10 , which can be generated by wrapping the membrane over the corresponding $T^{2}$, one readily finds the following expression for the most general scalar central charge ( $\sigma_{1,3}$ are the real Pauli matrices and the indices $i, j=1,2$ label the two supersymmetries),

$$
Z^{i j}=Z_{910} \delta^{i j}-\left(P_{9} \sigma_{3}^{i j}-P_{10} \sigma_{1}^{i j}\right)
$$


This result is, of course, in agreement with the $d=9$ entry of table 1 in section 1 . Here $P_{9}$ and $P_{10}$ denote the Kaluza-Klein momenta, while $Z_{910}$ is the winding number of the membrane on $T^{2}$. Assuming that we are compactifying over a torus with modular parameter $\tau$ and area $A$, the mass formula takes the form

$$
\begin{aligned}
M_{\mathrm{BPS}} & =\sqrt{P_{9}^{2}+P_{10}^{2}}+\left|Z_{910}\right| \\
& =\frac{1}{\sqrt{A \tau_{2}}}\left|q_{1}+\tau q_{2}\right|+T_{\mathrm{m}} A|p| .
\end{aligned}
$$

Here $q_{1,2}$ denote the momentum numbers on the torus and $p$ is the number of times the membrane is wrapped over torus; $T_{\mathrm{m}}$ denotes the supermembrane tension. Clearly, the KKA states correspond to the momentum modes while the KKB states are associated with the wrapped membranes on $T^{2}$. Hence there is a rather natural way to describe the IIA and IIB momentum and winding states starting from a (super)membrane in 11 spacetime dimensions [25, 27].

To really construct the BPS-extended supergravity theory associated with the supermembrane compactified on $T^{2}$, one would have to consider $N=2$ supergravity in nine spacetime dimensions and couple it to the simplest BPS supermultiplets corresponding to the KKA and KKB states. Nine-dimensional supergravity has precisely three gauge fields that couple to the three central charges discussed above. From the perspective of 11-dimensional supergravity compactified on $T^{2}$ the KKA multiplets are the KaluzaKlein states. Their charges transform obviously with respect to an $S O(2)$ associated with rotations of the coordinates labelled by 9 and 10 . Hence we have a double tower of these charges with corresponding KKA supermultiplets. On the other hand, from a IIB perspective, compactified on $S^{1}$, the KKB states are the Kaluza-Klein states and their charge is $S O(2)$ invariant. Here we have a single tower of $\mathrm{KKB}$ supermultiplets. From the perspective of nine-dimensional BPS-extended supergravity one should be able to couple both towers of KKA and KKB supermultiplets simultaneously, thereby arriving at a theory that contains 10-dimensional IIA and IIB theories in certain decompactification limits, as well as 11-dimensional supergravity. The BPS-extended theory is in some sense truly 12dimensional with three compact coordinates, although there is no 12-dimensional Lorentz invariance, not even in a uniform decompactification limit, as the fields never depend on all 12 coordinates! Whether this kind of BPS-extended supergravity offers a viable scheme in a more general context than the one we discuss here, remains to be seen. Very little work has been done in incorporating BPS and/or Kaluza-Klein multiplets into the field theory. Nevertheless, in the case at hand we know a lot about these couplings from our knowledge of the $T^{2}$ compactification of 11-dimensional supergravity and the $S^{1}$ compactification of IIB supergravity.

For the convenience of the reader we have listed the fields of nine-dimensional $N=2$ supergravity listed in table 1 , where we also indicate their relation with the fields of 11 dimensional and 10-dimensional IIA/B supergravity upon dimensional reduction. It is not necessary to work out all the nonlinear field redefinitions here, as the corresponding fields can be uniquely identified by their scaling weights under $S O(1,1)$, a symmetry of the massless theory that emerges upon dimensional reduction and is associated with scalings of the internal vielbeine.

Of particular relevance are the three Abelian vector gauge fields. There are the two vector fields $A_{\mu}^{\alpha}$, which are the Kaluza-Klein photons of the $T^{2}$ reduction of 11-dimensional supergravity and which couple therefore to the KKA states. From the IIA perspective these correspond to the Kaluza-Klein states on $S^{1}$ and the D0 states. From the IIB side they originate 
Table 3. The bosonic fields of the 11-dimensional, type-IIA, nine-dimensional $N=2$ and type-IIB supergravity theories. The 11-dimensional and 10-dimensional indices, respectively, are split as $\hat{M}=(\mu, 9,10)$ and $M=(\mu, 9)$, where $\mu=0,1, \ldots, 8$. The final column lists the $S O(1,1)$ scaling weights of the fields.

\begin{tabular}{lllll}
\hline$D=11$ & IIA & $D=9$ & IIB & $S O(1,1)$ \\
\hline$\hat{G}_{\mu \nu}$ & $G_{\mu \nu}$ & $g_{\mu \nu}$ & $G_{\mu \nu}$ & 0 \\
$\hat{A}_{\mu 910}$ & $C_{\mu 9}$ & $B_{\mu}$ & $G_{\mu 9}$ & -4 \\
$\hat{G}_{\mu 9}, \hat{G}_{\mu 10}$ & $G_{\mu 9}, C_{\mu}$ & $A_{\mu}^{\alpha}$ & $A_{\mu 9}^{\alpha}$ & 3 \\
$\hat{A}_{\mu \nu 9}, \hat{A}_{\mu \nu 10}$ & $C_{\mu \nu 9}, C_{\mu \nu}$ & $A_{\mu \nu}^{\alpha}$ & $A_{\mu \nu}^{\alpha}$ & -1 \\
$\hat{A}_{\mu \nu \rho}$ & $C_{\mu \nu \rho}$ & $A_{\mu \nu \rho}$ & $A_{\mu \nu \rho \sigma}$ & 2 \\
$\hat{G}_{910}, \hat{G}_{99}, \hat{G}_{1010}$ & $\phi, G_{99}, C_{9}$ & $\left\{\begin{array}{l}\phi^{\alpha} \\
\exp (\sigma)\end{array}\right.$ & $\phi^{\alpha}$ & 0 \\
\hline
\end{tabular}

from the tensor fields, which confirms that they couple to the IIB (elementary and D1) winding states. These two fields transform under $S L(2)$, which can be understood from the perspective of the modular transformation on $T^{2}$ as well as of the $S$-duality transformations that rotate the elementary with the D1 strings. The third gauge field, denoted by $B_{\mu}$, is an $S L(2)$ singlet and is the Kaluza-Klein photon on the IIB side, so that it couples to the KKB states. On the IIA side it originates from the IIA tensor field, which is consistent with the fact that the IIA winding states constitute KKB supermultiplets.

The resulting BPS-extended theory incorporates 11-dimensional supergravity and the two type-II supergravities in special decompactification limits. However, as we stressed above, we are dealing with a 12-dimensional theory here, of which three coordinates are compact, except that no field can depend on all three compact coordinates. The theory has obviously two mass scales associated with the KKA and KKB states. We return to them momentarily. Both $S$ and $T$-duality are manifest, although the latter has become trivial as the theory is not based on a specific IIA or IIB perspective. One has the freedom to view the theory from a IIA or a IIB perspective and interpret it accordingly.

We should discuss the fate of the group $G=S O(1,1) \times S L(2, \mathbb{R})$ of pure supergravity after coupling the theory to BPS multiplets. The central charges of the Kaluza-Klein states form a discrete lattice, which is affected by this group. Hence, after coupling to the BPS states, we only have a discrete subgroup that leaves the charge lattice invariant. This is the group $S L(2, Z)$.

The KKA and KKB states and their interactions can be understood from the 11dimensional and IIB supergravity perspective. Therefore, we can deduce the following BPS mass formula:

$$
M_{\mathrm{BPS}}\left(q_{1}, q_{2}, p\right)=m_{\mathrm{KKA}} \mathrm{e}^{3 \sigma / 7}\left|q_{\alpha} \phi^{\alpha}\right|+m_{\mathrm{KKB}} \mathrm{e}^{-4 \sigma / 7}|p|,
$$

where $q_{\alpha}$ and $p$ refer to the integer-valued KKA and KKB charges, respectively, and $m_{\mathrm{KKA}}$ and $m_{\mathrm{KKB}}$ are two independent mass scales. This formula can be compared with the membrane BPS formula (36) in the 11-dimensional frame. One then finds that

$$
m_{\mathrm{KKA}}^{2} m_{\mathrm{KKB}} \propto T_{\mathrm{m}},
$$

without field-dependent factors. 
The above example of a BPS-extended supergravity theory shows that one obtains a dichotomic theory which can be regarded as a 12-dimensional field theory. Various decompactification limits correspond to 11-dimensional supergravity or 10-dimensional IIA/B supergravity. There are interesting questions regarding the field-theoretic coupling of the fields associated with the BPS states. In the case at hand these can, in principle, be answered, because the couplings can be deduced from the coupling of the massive Kaluza-Klein fields in the compactifications of 11-dimensional and IIB supergravity. One such questions concerns the role of the local symmetry $H=S O(2)$ that one uses in the description of the $S L(2) / S O(2)$ coset space for the nonlinear sigma model. There is a composite gauge field associated with the group $S O(2)$, which does not correspond to additional degrees of freedom. To study this aspect in a little more detail, let us consider a simplified example (worked out in collaboration with I Herger) illustrating the action of the hidden symmetries when the massive Kaluza-Klein modes are retained, namely a nonlinear sigma model based on the coset space $S L(n, \mathbb{R}) / S O(n)$ in flat spacetime. In the following, we will split the higher-dimensional coordinates as $z^{M}=\left(x^{\mu}, y^{m}\right)$, where the $y^{m}$ parametrize a torus. The degrees of freedom are thus contained in a matrix $\mathcal{V}(x, y) \in S L(n, \mathbb{R})$ transforming as

$$
\mathcal{V}(x, y) \rightarrow g \mathcal{V}(x, y) h^{-1}(x, y),
$$

where $g$ denotes a (constant) element of $S L(n, \mathbb{R})$ and $h(x, y)$ is a local $S O(n)$ transformation. In view of the gauge invariance that depends on both $x$ and $y$, and the fact that we are dealing with a group element, the split into massive and massless degrees of freedom is not entirely straightforward.

The best approach is to write $\mathcal{V}(x, y)$ as the product of two $S L(n, \mathbb{R})$ elements,

$$
\mathcal{V}(x, y)=\mathcal{V}_{0}(x) \mathcal{V}_{1}(x, y)
$$

and to require that $\mathcal{V}_{0}$ describes the massless modes in the torus compactification. To do this, one can first fix the $S O(n)$ gauge freedom and define a coset representative. Subsequently, one considers the logarithm of $\mathcal{V}(x, y)$ and expands it in terms of Fourier modes on the torus. Dropping the $y$-dependent modes in this expansion yields $\mathcal{V}_{0}(x)$. However, $\mathcal{V}_{0}(x)$ is itself a coset representative so that it is defined up to multiplication by an $x$-dependent $S O(n)$ transformation acting from the right. This leads to a corresponding ambiguity for $\mathcal{V}_{1}(x, y)$. Hence $\mathcal{V}_{0}(x)$ parametrizes a nonlinear sigma model in the lower-dimensional space, so that it transforms according to

$$
\mathcal{V}_{0}(x) \rightarrow g \mathcal{V}_{0}(x) h_{0}^{-1}(x),
$$

where $h_{0}(x)$ is an $x$-dependent $S O(n)$ transformation, and $\mathcal{V}_{1}(x, y)$ transforms under an $x$ dependent $S O(n)$ transformation from the left and, provided one again relaxes the original gauge condition, under an $x$ - and $y$-dependent $S O(n)$ transformation from the right,

$$
\mathcal{V}_{1}(x, y) \rightarrow h_{0}(x) \mathcal{V}_{1}(x, y) h_{1}^{-1}(x, y) h_{0}^{-1}(x),
$$

where we have defined $h(x, y)=h_{0}(x) h_{1}(x, y)$. All the massive Kaluza-Klein degrees of freedom thus reside in $\mathcal{V}_{1}(x, y)$. The $S O(n)$ symmetry corresponding to $h_{1}(x, y)$ can now be fixed by going to a 'unitary gauge',

$$
\mathcal{V}_{1}(x, y)=\exp \phi(x, y),
$$


where $\phi(x, y)$ is a symmetric traceless $n \times n$ matrix, such that $\mathcal{V}_{1}(x, y)$ transforms under the residual $x$-dependent $S O(n)$ transformations according to

$\mathcal{V}_{1}(x, y) \rightarrow h_{0}(x) \mathcal{V}_{1}(x, y) h_{0}^{-1}(x), \quad \phi(x, y) \rightarrow h_{0}(x) \phi(x, y) h_{0}^{-1}(x)$.

Thus the massive fields $\phi(x, y)$ transform covariantly under $x$-dependent $S O(n)$ gauge transformations but not under $S L(n, \mathbb{R})$.

The split (40) of $\mathcal{V}(x, y)$ exhibits clearly how the massive Kaluza-Klein degrees of freedom behave with respect to the local symmetries of the massless theory. To describe the Lagrangian we consider the $S L(n, \mathbb{R})$ Lie-algebra-valued expression

$$
\begin{aligned}
P_{M}+Q_{M} & :=\mathcal{V}^{-1} \partial_{M} \mathcal{V} \\
& =\mathcal{V}_{1}^{-1} P_{M}^{0} \mathcal{V}_{1}+\mathcal{V}_{1}^{-1} D_{M}^{0} \mathcal{V}_{1}+Q_{M}^{0},
\end{aligned}
$$

where $Q_{M}$ and $P_{M}$ belong to the Lie algebra of $S O(n)$ and its complement, respectively, in the Lie algebra of $S L(n, \mathbb{R})$. Splitting the index $M$ into $\mu$ and $m$ as before, we have the $y$-independent quantities

$$
P_{\mu}^{0}+Q_{\mu}^{0}:=\mathcal{V}_{0}^{-1} \partial_{\mu} \mathcal{V}_{0}
$$

(obviously, $Q_{m}^{0}=P_{m}^{0}=0$ ). The derivative $D_{M}^{0}$ is covariant with respect to $x$-dependent $S O(n)$ gauge transformations,

$$
D_{\mu}^{0} \mathcal{V}_{1}:=\partial_{\mu} \mathcal{V}_{1}+\left[Q_{\mu}^{0}, \mathcal{V}_{1}\right], \quad D_{m}^{0} \mathcal{V}_{1}:=\partial_{m} \mathcal{V}_{1}
$$

To write down an action coupling the massless sector and the massive Kaluza-Klein modes in an $S O(n)$ invariant way, we expand

$$
P_{M}=P_{M}^{0}+D_{M}^{0} \phi+\left[P_{M}^{0}, \phi\right]+\frac{1}{2}\left[\left[P_{M}^{0}, \phi\right], \phi\right]+\frac{1}{2}\left[D_{M}^{0} \phi, \phi\right]+\cdots,
$$

projected on the complement of the Lie algebra of $S O(n)$. Because the $S L(n, \mathbb{R}) / S O(n)$ coset space is symmetric some of the terms in $P_{M}$ will vanish trivially. What remains is to substitute the expression for $P_{M}$ into

$$
L=-\frac{1}{2} \operatorname{Tr}\left(P_{\mu}^{2}\right)-\frac{1}{2} \operatorname{Tr}\left(P_{m}^{2}\right),
$$

which will lead to an action that is non-polynomial in $\phi$. Let us repeat, however, that this action is invariant under $x$-dependent gauge transformations, as well as under a global $S L(n, \mathbb{R})$ symmetry which acts exclusively in the massless sector. Once we fix an $S O(n)$ gauge, the $S L(n, \mathbb{R})$ symmetry becomes nonlinearly realized and acts also on the massive fields.

Before fixing an $S O(n)$ gauge, the $S L(n, \mathbb{R})$ symmetry does not act on the massive modes in this simplified model. This is not so when the $S L(n, \mathbb{R})$ originates from the dimensional reduction in the more complicated models based on (super)gravity in higher dimensions. Upon performing a Kaluza-Klein reduction (not a truncation!) on the torus $T^{n}$, the global symmetry will still act on the massive modes, but it will be broken to an arithmetic subgroup such as $G(Z)$. To see how this comes about, recall that $G=S L(n, \mathbb{R})$ and $H=S O(n)$ are precisely the symmetries that one obtains upon dimensional reduction of pure Einstein theory on a torus $T^{n}$. As we saw in the foregoing section, the Kaluza-Klein gauge field $B_{\mu}{ }^{m}$ couples via the derivative operator (14),

$$
\mathcal{D}_{\mu}=\partial_{\mu}-B_{\mu}{ }^{m} \partial_{m}
$$

(with vanishing affine connection for the torus). When the theory is compactified on a torus $T^{n}$, the derivative operator $\partial_{m}$ will only admit discrete eigenvalues $\boldsymbol{q}=\left(q_{1}, \ldots, q_{m}\right)$. These 
eigenvalues lie on an $n$-dimensional lattice, the lattice of Kaluza-Klein charges. It is the presence of this lattice that leads to the breaking $S L(n, \mathbb{R}) \rightarrow S L(n, Z)$ : the group $S L(n, Z)$ acts on the vectors $\boldsymbol{q}$ labelling the Kaluza-Klein modes, rather than on the fields themselves. The massless modes have $\boldsymbol{q}=0$ and transform under $S L(n, Z)$ in the way described above for the nonlinear sigma model.

Further results along these lines will be published elsewhere.

\section{Acknowledgments}

The Kaluza-Klein reduction of nonlinear sigma models was obtained in collaboration with Ivan Herger. We thank the organizers of the Gürsey Memorial Conference for inviting us to a pleasant and stimulating meeting. This work is supported in part by the European Commission RTN programmes HPRN-CT-2000-00122 and HPRN-CT-2000-00131 (in the latter HN is associated with U Bonn).

\section{References}

[1] Cremmer E, Julia B and Scherk J 1978 Phys. Lett. B 76409

[2] Hull C M and Townsend P K 1995 Nucl. Phys. B 438109 (Hull C M and Townsend P K 1994 Preprint hep-th/9410167)

[3] Townsend P K 1995 Phys. Lett. B 350184 (Townsend P K 1995 Preprint hep-th/9501068)

[4] Witten E 1995 Nucl. Phys. B 44385 (Witten E 1995 Preprint hep-th/9503124)

[5] Cremmer E and Julia B 1978 Phys. Lett. B 8048 Cremmer E and Julia B 1979 Nucl. Phys. B 159141

[6] de Wit B and Nicolai H 1985 Phys. Lett. B 15547 de Wit B and Nicolai H 1986 Nucl. Phys. B 274363

[7] Nicolai H 1987 Phys. Lett. B 187363

[8] de Wit B 2001 Int. J. Mod. Phys. A 161002 (de Wit B 2000 Preprint hep-th/0010292)

[9] de Wit B and Louis J 1999 Strings, branes and dualities Proc. NATO Advanced Study Institute (Cargèse, 1997) NATO ASI C520 ed L Baulieu, P Di Francesco, M Douglas, V Kazakov, M Picco and P Windey (Dordrecht: Kluwer) p 33 (de Wit B and Louis J 1998 Preprint hep-th/9801132)

[10] Obers N A and Pioline B 1999 Phys. Rep. 318113 (Obers N A and Pioline B 1998 Preprint hep-th/9809039)

[11] Koepsell K, Nicolai H and Samtleben H A J 1999 J. High Energy Phys. JHEP04(1999)023

[12] Koepsell K, Nicolai H and Samtleben H A J 2000 Class. Quantum Grav. 173689

[13] Cvetic M, Lu H and Pope C N 2000 Nucl. Phys. B 584149 (Cvetic M, Lu H and Pope C N 2000 Preprint hep-th/0002099) Pilch K and Warner N P 2000 Phys. Lett. B 48722 (Pilch K and Warner N P 2000 Preprint hep-th/0002192)

[14] Ashtekar A 1986 Phys. Rev. Lett. 572244

[15] Melosch S and Nicolai H 1998 Phys. Lett. B 41691

[16] Julia B 1983 Unified Field Theories in More than 4 Dimensions ed V D Sabbata and E Schmutzer (Singapore: World Scientific)

[17] Marcus N and Schwarz J H 1983 Nucl. Phys. B 228145

[18] de Wit B and Nicolai H 1987 Nucl. Phys. B 281211

[19] Nastase H, Vaman D and van Nieuwenhuizen P 2000 Nucl. Phys. B 581179

[20] de Wit B and Nicolai H 1982 Nucl. Phys. B 208323

[21] de Wit B and Nicolai H 1984 Nucl. Phys. B 231506

[22] Günaydin M, Romans L and Warner N P 1986 Nucl. Phys. B 272598

[23] Nicolai H and Samtleben H A J 2001 Phys. Rev. Lett. 861686 (Nicolai H and Samtleben H A J 2000 Preprint hep-th/0010076) 
[24] Sohnius M F 1978 Nucl. Phys. B 138109

[25] Abou-Zeid M, de Wit B, Lüst D and Nicolai H 1999 Phys. Lett. B 466144

[26] Bergshoeff E, Sezgin E and Townsend P K 1987 Phys. Lett. B 18975

Bergshoeff E, Sezgin E and Townsend P K 1988 Ann. Phys. 185330

[27] Schwarz J H 1996 Phys. Lett. B 36797

(Schwarz J H 1995 Preprint hep-th/9510086)

Aspinwall P S 1996 Nucl. Phys. Proc. Suppl. 4630

(Aspinwall P S 1995 Preprint hep-th/9508154) 